\title{
Challenges posed by novel vaccines in a hyperlinked society both to doctors and the health of their patients
}

\begin{abstract}
ALOJZ IHAN
Abstract Medicine in the developed world is becoming progressively more commercialized since patients (or, more accurately, medical consumers) are increasingly becoming the driving force of what we call medical progress. The doctors are primarily obliged to treat and help patients, but will always have also (commercial) temptation before him: to facilitate the consumer's desires and, with a warm heart, shoot to the far borders of medical knowledge and technology. A good example illustrating dilemma of beneficial vs commercial medicine are ethical challenges of some new vaccines developed recently. Since their earliest introduction in the late 18th century, vaccines have undoubtedly saved the lives of millions, while also fundamentally changing the way modern medicine is practiced. Many diseases that were once widespread are now eradicated, yet vaccine development faces ongoing challenges, including some important ethical considerations. Today there are over 300 vaccines in development and included in clinical trials. Among them, anti-addiction vaccines (Cocaine, Nicotine, Fentanyl, Heroin, Oxycodone) received a burst of media attention, because these vaccines could help people in recovery from addiction; however, many ethical and legal questions may arise before clinical use.
\end{abstract}

Keywords: $\bullet$ conflicts of interest $\bullet$ physicians $\bullet$ commercialization $\bullet$ populism - public health $\bullet$ vaccine development $\bullet$ anti-addiction vaccines $\bullet$ vaccines for pregnant mothers $\bullet$ ethical and legal questions •

CORRESPONDENCE AdDRESs: Alojz Ihan, dr. med., Ph.D., Full Professor, University of Ljubljana, Faculty of Medicine, Institut of Microbiology and Immunology, Zaloška 4,1000 Ljubljana Slovenia, e-mail: alojz.ihan@mf.uni-lj.si.

DOI https://doi.org/10.18690/2463-7955.11.1.1-12.(2018)

ISSN 2463-7955

(C) 2018 University of Maribor Press

Available at http://journals.um.si/ 
It is difficult to predict what the future holds in terms of our health and the possibility that individuals will one day be able to care for their own health according to their own judgment, thus avoiding disease. The main problem, of course, is simply the fact that the rhythm of medical discoveries and changes can only be observed over the course of a short period. In the past thousand years of medicine, there have been many undulations. But even when trying to prophesize over shorter time frame, for example a few decades, it might be instructive to look back a hundred years and wonder what a doctor could have predicted about the progress of medicine during the next century, which leads us to the present. Indeed, we must admit that the predictions of our medical colleagues from the previous century could contain very little of what was actually dreamed of and achieved by twentieth century doctors. This is simply because much of the diagnostic and treatment methods that are absolutely normal for today's doctors and patients did not figure into even the wildest imaginations a hundred years ago. I need only mention the treatment of infectious diseases with antibiotics, cancer with chemotherapy and radiation therapy, anesthetic techniques and other technologies that allow long-lasting surgical interventions and transplants among others. Diagnostic techniques at the beginning of the twentieth century did not contain a hint of the enzyme, immune, genetic tests that today provide us with an enormous amount of data regarding the cause of medical difficulties. Of course there were also no diagnostic technologies from magnetic resonance imaging to ultrasounds that, with computer imaging, optic cables, lasers and all of their mysterious brilliance, which cast a spell over doctor and patient alike (Speed, 2017).

Indeed, no writer a hundred years ago of any kind could possibly have predicted the development of medicine. This is especially true if we think of medical progress as little more than a series of a collection of medical technologies that during a given period happen to inspire the most trust in both patients and doctors. In the coming century, medical progress will, if anything, be even more rapid. Each new innovation which will promise better diagnostic and therapeutic possibilities, (even if it is only a whisper of a promise!) will be subject to ever more effective marketing methods, quickly becoming available to any doctor or patient in the developed world. Here, I emphasize the word »patient «. The fundamental issue here is, of course, that medicine in the developed world is becoming more and more commercialized. Pharmaceutical companies and other players in the health-care industry will strive to sell as many products as possible. For this reason, advertising and the media »cultivate « (potential) patients, urging them to become increasingly capricious in the sense of health creation through consumption. I am thinking of the tens and hundreds of »home « remedies that line the shelves of drug stores, pharmacies and health food stores beckoning to the growing circle of buyers undergoing their own therapeutic experiments. Many people today, even as we stand on the threshold of a »new hyperlinked (health) informational era«, spend as 
much time studying and testing all these new preparations as they should on cultivating good relations with their life partners and their children. The latter would, of course, bring them a great deal more health and satisfaction than childishly wallowing in obsession with their own supposed »sickness «. It is possible to conclude from these observations that the producers of one of the greatest "negative" pressures in the medical paradigm (again in the developed world) are the pharmaceutical giants who view the neurotic hypochondriac, hyper informed and hyperlinked "patient" as the ideal and insatiable medical consumer. These are people who, with little knowledge but great self-assurance, rummage through internet sale catalogues and order medical preparations for enhanced health and protection against various (more or less imagined) ailments (Perkmann, 2013).

Despite the fact that this nearly infinite array of home remedies, of vitamins and herbal preparations, is fairly innocent from the medical perspective, yet from the cultural point of view it tends to steer the attention of the lay (non-medical) public from their own health to their own (potential or actual) sickness. This is a crucial difference! It takes us away from the role for which we are entirely competent (cultivation of the well-being and happiness that also brings health) and pushes us into a role for which most of us are neither educated nor capable (efforts to discover, prevent and treat illness). And what, in order to stimulate unfettered consumption, is more suitable than people who are, on the one hand, diverted from their own feelings (characterized by personal health and energy) and, on the other hand, submerged in a sea of medical diagnostics, terminology and therapies over which they have neither overview or detailed understanding?

Of course, it is only to be expected that the pharmaceutical industry will direct its energies to where there is profit. Industry is measured by profit. However, if we want to focus our attention on our health (as opposed to our sickness), then we must try to live in the greatest possible harmony with ourselves and the environment, through cultivating satisfying, interpersonal relationships and by avoiding unhealthy life styles (stress, dependency, overwork, one-sided response to stimuli, environmental hazards). But all of this will not bring much in the way of profits to the pharmaceutical industry. Naturally, it is not terribly enthusiastic about this vision of the (non)consumer. The industry as a whole would certainly be much more attracted to the neurotic who is constantly fretting over all the illnesses that are (potentially) threatening and all of the different colored pills that might offer some protection (Hawkins et al., 2012). That is the ideal consumer for the pharmaceutical industry, its golden calf (Nestle, 2016).

\section{Do doctors care about patient or medical consumers}

And what about doctors? The tendency to cultivate increasingly demanding (and capricious) medical consumers would hardly seem to suit the »old-fashioned « image of the doctor. At least not in the first instance when the doctor sees the 
patient's self-healing theories and practices as resistance and doubts in the doctor's own professional authority and judgment. But after the passage of time, after the first shock has passed, the doctor may come to the pragmatic realization that a patient transformed into a medical consumer, though admittedly more difficult and demanding, offers an attractive niche in the market, not only to the pharmaceutical industry, but also a business challenge to the doctor. Why indeed would the doctor complain in the face of a new, exciting (and even profitable) challenge? If the patient, in his or her confusion, begins to conflate all of life's problems with the body and illness, the doctor can say, without a bad conscious, and in the spirit of the times that the patient (the customer) is king and that the patient's desire is the doctor's command. It is not even necessary to address the issue of profits. Of course, the doctor could tell the patient what he really thinks of "symptoms « and »diagnoses «. But in today's free market conditions, this might mean that the patient would seek a second opinion. On the other hand, the doctor can try to hunt down real medical causes for various troubles, but that is generally complicated and takes up time, and sometimes even the professional capability of the doctor. In any case, most patients simply want pills from their doctors and have no desire to face life's problems and find real solutions for them (either with or without the help of a doctor). And if it seems too difficult for the patient to deal with life's real problems, and he or she would prefer to focus on »consumer « substitutes, why indeed should the doctor force the patient involuntarily into such much-needed soul searching?

Most of them do not. After the initial phase of complaining about demanding and capricious patients, it becomes clear to the doctor that the pharmaceutical industry has actually done him a favor, what with all of the advertising and the multi-vitamin preparations, the way has been paved, if not for an ideal doctor-patient relationship, at least for a profitable one.

But where is the border when medicine in pursuit of profit ceases to be beneficial to the patient? Unfortunately, it will never be easy to make a clear dividing line between commercial and beneficial medicine; it has to be found in each individual case. It is not possible to establish once and for all that liposuction, for example, is an unneeded medical intervention. Yet on the other hand, it is also unrealistic to claim that it is always justified or a legitimate medical intervention (even when the patient wants it). The same logic applies to countless other medical procedures and treatments. The doctor will always have the (commercial) temptation before him: to facilitate the consumer's desires and, with a warm, optimistic smile, shoot to the far borders of medical knowledge and technology. Yet the doctor as a professional should also realize when the patient's desires move in the direction of returning to a healthy life balance, and when they merely point the way towards a fairy-tale project that has nothing to do with the state of his or her health. If anything this is the contrary. The dividing line between commercial and beneficial medicine is namely the dividing line between a doctor's actions that bring the patient closer to both sensing and feeling responsible for his or her own health, and a doctor's actions 
which turns the patient away from his or her sensations into the confused, incomprehensible world of diagnostics and magically therapeutic procedures (Anderson, 2014).

The next decades of medicine will therefore be a period when it becomes necessary to define the dividing line between beneficial medicine and medicine that serves only the interest of the consumer, providing balm for hysterical, unfulfilled human needs. This dividing line will become even more difficult to define as nearly all the developed countries have expressed the intention to shift financing the health bill, as much as possible, from state (restricted) accounts to private (insurance) coffers. Of course, this is the easiest and least responsible defense of the government bureaucracies against the medical consumer. Yet they are forgetting that simply shifting the financing will not eliminate the growth of medical consumption. On the contrary, the national governments, because of their retreat from financing medical expenses, will become less interested in organizing a rational medical cost structure. The emancipated insurers will do business in the way all insurance companies do in accordance with the free market and driven by profits, but without any long-term interest in controlling the amount of money that flows into the medical system from the pockets of the insured. In regards to physicians, it is not clear to what an extent that they, in the dual role of doctors and businessmen, will pursue the goals of beneficial medicine, resisting the motivation of medical consumerism. If the patient/consumer wants a therapy that is useless, but at the same time harmless, and, on top of that, brings profit to the doctor, it is not realistic to expect that the doctor/businessman will attempt to persuade the patient of this brave new century to give up his or her unrealistic medical desires.

Thus the coming decades will bring many challenges to what Hippocrates, the father of modern medicine, characterized as the basic relationship between illness and health. Hippocrates was responsible for driving away the magic medicine men who danced before the patient, orchestrated the burning of a sacrifice, spoke in tongues to one another divinity, took the patient's money and departed. Hippocrates also resisted the banal commercialization taking place in the medical circles of ancient Greece insofar as he demanded a detailed, written description of the patient's condition and system before and after the healing process. In such a way, it would be clearly recorded whether the doctor's treatment helped the patient or whether it was mere torment with no real medical benefit. Though it may seem paradoxical, it seems that Hippocrates' demand for beneficial medicine will become important once again. (Studdert et al., 2004).

\section{$3 \quad$ New vaccines, new ethical and legal questions}

In the past, vaccines have undoubtedly saved the lives of millions, and many diseases that were once widespread are now eradicated. However, new vaccine development faces ongoing challenges, including some important ethical 
controversies. Ethical challenges related to vaccine development mostly revolve around research and testing, but also evidence of safety and efficacy regarding longterm health. For example, anti-addiction vaccines (Cocaine, Nicotine, Fentanyl, Heroin, Oxycodone) received a burst of media attention, since these vaccines could help people in recovery from addiction. However, many ethical and legal questions, including human rights and personal freedom, may arise before clinical use. Undoubtedly drug addiction is a serious medical disease with side-effects that represent a serious public health threat. Since vaccination has historically proven to be a very effective method of combating disease, vaccination against additive drugs may be an effective method of protecting the public welfare (Haney et al., 2010). However, an argument against antidrug vaccinations may be the preservation of personal freedom for certain behavioral choices. It could be argued that preventive use of vaccines violates the minor's right to express their free will. On the other hand, such an argument is pretty similar to those raised by parents who claim vaccination requirements usurp their due process rights to make parental decisions on behalf of their children (Shen et al., 2011).

Alexis Osburn published (Osburn, 2008) an interesting text "The Argument for Incorporating Emerging Anti-Addiction Vaccines into Existing Compulsory Immunization Statutes", with a systematic analysis of six factors concerning human rights to be satisfied before a vaccine is incorporated into a state's vaccination statute:

1) the danger to public health must be substantial,

2) the condition must have serious consequences if transmitted,

3 ) the effectiveness of the vaccine in safeguarding the majority of the public against the particular malady must be well established,

4) the vaccine must be the most appropriate, least invasive, and most conservative means of achieving the desired public health objective,

5) the individual must be provided with an appreciable benefit not dependent on speculation about hypothetical future behaviors, and

6) the burden to the individual's human rights must be balanced against, and found to be substantially outweighed by, the benefit to society in helping prevent a highly contagious disease or other potentially calamitous condition from affecting the public health.

In addition, the preventive use of an anti-addiction vaccine in healthy young people requires stronger evidence of safety and efficacy than shorter-term use to reduce relapse in adults who are drug dependent. Unfortunately, obtaining evidence to meet regulatory requirements for such use is very expensive. Another side effect of antiaddiction vaccines arises from the fact that vaccines only produce anti-drug antibodies for drug neutralization; however the drug dependency remains. The smoker, vaccinated by anti-nicotine vaccine, may be urged to smoke ten times more cigarettes in order to overcome the nicotine blockade; the result of such high levels 
of smoking for prolonged periods would be a massive increase of ingested carcinogens, which the vaccine does not block. Finally, parents might request that their children be vaccinated to prevent future drug abuse, even though the risks of future drug abuse for them as individuals might not be well defined (Shen et al., 2011).

The proof of principle for an anti-addiction vaccine was first demonstrated by two studies. Berkowitz and colleagues (Berkowitz and Spector, 1972) published their creation of a morphine vaccine in animals. Using rats, they administered a morphine hapten linked to bovine serum albumin (BSA, a carrier protein) and created antimorphine antibodies. These antibodies reduced the concentration of free morphine in the plasma of their vaccinated rats. Bonese et. al. (Bonese et al., 1974) later created a similar vaccine in primates, in which the vaccinated rhesus monkey primates decreased their self-administration of heroin (Volkow and Collins, 2017).

The data from cocaine and nicotine vaccine trials suggest that many patients may not produce a sufficient antibody response for clinical efficacy, but those patients who do attain high levels of antibodies are helped to remain abstinent (Haney et al., 2010). If extending this technology to other abused substances is successful, it will be a tremendous benefit to have innovative pharmacotherapies rapidly available, especially as new "designer drugs" are introduced. Indeed, many anti-addiction vaccines are in various stages of development for quite a broad array of abused drugs, including cocaine, nicotine, methamphetamine, and heroin (Polosa, 2011).

Vaccine efficacy depends on many critical factors, such as antibody specificity, affinity, and antibody concentration (titer), which are affected by the design of the vaccine conjugate, the dose of the vaccine, the adjuvant selection, the frequency of vaccinations, the time interval between immunizations, and maybe the poorly understood genetic variations among individuals (Shen et al., 2011).

The idea of vaccinating people against drugs of abuse is decades old. Researchers have repeatedly validated the concept in animals but have yet to demonstrate a vaccine that is effective in people (Stowe et al., 2011).

Learning from the failures of past vaccines, scientists in the field have now developed new formulations that are closing in on or have already begun clinical trials. Antidrug vaccines are created by the synthesis of a chemical derivative of the drug (hapten) attached to a highly immunogenic foreign protein (carrier) through a chemical linker (adjuvants) to induce high levels and long duration of antibodies against a specific drug (Shen et al., 2011). 
A. Ihan: Challenges posed by novel vaccines in a hyperlinked society both to doctors and the health of their patients

\section{Development of vaccines for pregnant women in order to protect the newborn}

Immunization of pregnant women ("maternal immunization") has the potential to protect mothers, fetuses, and infants from infectious diseases, as well as to prevent complications due to maternal infection during prenatal development. However, there are relatively few clinical trials or prospective studies that include pregnant women. Pregnant women and infants are generally classified as vulnerable subjects, and because of that are often excluded from clinical trials. Investigators are often reluctant to include pregnant women, as there are additional health concerns, and a need to avoid unnecessary risk to the fetus. Informing researchers of the ethics of including vulnerable subjects in controlled trials may increase willingness to include pregnant women and infants in safety studies (Beelera, 2016).

Currently only two vaccines are routinely recommended during pregnancy influenza and pertussis vaccine, in which the safety of both vaccines is excellent. Close household contacts such as siblings, partners and grandparents should also be up-to-date vaccinated with all of their age-appropriate vaccines, such as rotavirus, varicella and MMR, (measles-mumps rubella) and particularly pertussis vaccine (Leask, 2013).

Children tare vaccinated against pertussis with three doses of the vaccine during the first year after birth and with a fourth dose in the second. The first dose of the vaccine is given to our children at the age of three months but is protected only after the second or third dose of the vaccine. Since the child is not be protected by the vaccine before the second vaccination, the child maintains protection against the disease due to antibodies that he acquired during pregnancy from the blood of their protected mother. In recent years, doctors strongly recommended vaccination of pregnant women against pertussis, so that the children are protected in these first months until they are effectively vaccinated (Bremer, 2017).

Pertussis is a disease caused by bacteria Bordetella pertussis and particularly affects younger children. Vaccination effectively protects against such illness, but does not prevent persistence of bacteria from forming on the respiratory tract. That's why a vaccinated person can also keep the infection if obtained from a sick person for a few weeks on the respiratory mucous membrane and spread it to other people, even if he does not get sick. That's why the Whooping cough in Slovenia has never disappeared altogether. Despite a high percentage of vaccinated children, a few hundred people get sick each year (however thousands of people were affected before the introduction of vaccination). In very young children, this can be a very stressful and endangering disease, and the child may die or suffer permanent brain damage due to respiratory distress in the event of coughing (Regan, 2016). 
If a pregnant woman gets vaccinated, the antibodies that are formed after vaccination enter the child's blood circulation through the placenta and protect the newborn from infection in the first months after birth. The antibodies begin to pass through the placenta after the 16th week of pregnancy, and the optimal time for vaccination is as soon as possible after the 24 th week of pregnancy. Throughout the world, pregnant women are vaccinated with a combination vaccine against diphtheria, tetanus and pertussis. Vaccination is advisable in every pregnancy to protect newborns against pertussis.

Pregnant flu vaccines are also recommended for pregnant women, as they are among the groups where the flu is more difficult. This vaccination is carried out according to the season of the flu, most importantly administered in October and November, regardless of the period of pregnancy. Otherwise, vaccinations with live vaccines, such as measles, mumps and rubella vaccines, yellow fever and varicella, are not recommended during pregnancy due to poor immune response of the pregnant woman and immature immune response (Amirthalingam, 2014).

Development of vaccines for pregnant women against streptococcal sepsis of newborns

Invasive group B streptococcal (GBS) infection is a dominant cause of morbidity and mortality in neonates. More than 40 years ago it was described that a lifethreatening invasive SGB infection was more frequent in neonates of mothers who have low serum antibodies against SGB serotypes that have caused an invasive neonate disease (Baker, 1976). However, decades before the correlation between a low concentration of maternally derived antibody against GBS capsular polysaccharide (CPS) of type III group B Streptococcus and infant susceptibility to GBS type III infection was described, the first evidence of the protective nature of CPS-specific antibodies was published in the 1930s when Rebecca Lancefield demonstrated that, using CPS-specific polyclonal rabbit serum, mice could be protected against GBS infections. (Lancefield, 1934). In 1976 Baker and Kasper provided the proof of concept that maternal vaccination could be a suitable effective strategy to prevent GBS infection in newborns, supporting the rationale for the development of a vaccine against GBS using CPS (Baker, 1976).

Since the disease occurs in the early period of the newborn's life, it is more reasonable than vaccinating a newborn baby to vaccinate a pregnant woman whose protective IgG antibodies against SGB are then transported into the child's blood. The results of immunization of pregnant women with glycoconjugated vaccine preparations suggest that maternal antibodies against SGB in the newborn remain for 2 - 3 months. The major migration of antibodies to the newborn occurs after 33 weeks of gestation, so preterm infants born before 34 weeks of gestation have reduced level of protective antibodies despite vaccination (Heath, 2017). 
Polysaccharide vaccine against capsular polysaccharide (CPS) is the first to be tested against SGB since 1930. In order to improve immunogenicity, experimental glycoconjugated vaccines with CPS were later developed. In conjugated vaccines, the polysaccharide is covalently bound to the protein carrier. The proteinpolysaccharide conjugate is much more immunogenic than the polysaccharide itself. In experimental conjugated vaccines against SGB, tetanus toxoid (TT) and non-toxic mutated form of diphtheria toxin (CRM) were most commonly used as carriers to CPS proteins. Both carriers are also commonly known in conjugated vaccines, and with both carriers they achieved comparable immunogenic effects in SGB vaccine testing phase 2 (Verani, 2010).

The need for a strain specific CRM vaccine is another disadvantage of polysaccharide vaccines, in addition to their lower immunogenicity, which limits the effectiveness of the SGB vaccine. With this, the vaccine can quickly become ineffective when changing the SGB serotypes that prevail in the population. Therefore, the progress of analyses of the SGB genome enabled the development of SGB proteins that are common to all SGB types. With the technique of sequencing the entire bacterial genome (WGS), in 2015, MinervaX launched phase 1 of the clinical trial of a protein SGB vaccine based on the fusion of N-terminal parts of two surface proteins SGB, AlphaC and Rib (GBSNN) (NCT02459262) 259. MinervaX expects the GBS-NN antigen to be protected against 95\% of SGB strains (Di Renzo, 2015).

\section{Conclusions}

In the past, vaccines have undoubtedly saved the lives of millions, and many diseases that were once widespread are now eradicated. However, new vaccine development faces ongoing challenges, including some important ethical controversies. Ethical challenges mostly revolve around research and testing, but also evidence of safety and efficacy regarding long-term health (Beelera, 2016). Every new vaccine introduction should be systematically evaluated, as well as for ethical consequences, before a vaccine is incorporated into a vaccination program. The effectiveness of the vaccine in safeguarding the majority of the public against the particular malady must be well established and the vaccine must be the most appropriate, least invasive, and most conservative means of achieving the desired public health objective. The individual must be provided with an appreciable benefit and the burden to the individual's human rights must be substantially outweighed by the benefit to society. Considering that vaccination against drugs is not a vaccination to prevent an infectious disease, the preventive use of anti-addictive vaccination should be applied if there is strong evidence of safety and efficacy. In addition, it should be applied only after an accurate balancing of the different values to be assigned to individual and public interests. 
The results from human studies of the first cocaine vaccine and three nicotine vaccines are promising, and preclinical development of efficacious methamphetamine and opiate vaccines is rapidly progressing (Bremer, 2017). Blocking immediate behavioral and toxic drug effects is valuable, but even more promising from the addiction perspective is the inhibition of drug reinforcement, which will be necessary to help prevent relapse to drug use by individuals motivated to quit (Regan, 2016). Ethical, legal, and regulatory challenges are the barriers to implementation of anti-addition vaccines, and future development strategies may include examining additional ways to increase antibody levels in high proportions of immunized individuals to improve vaccine efficacy. Advances in vaccine conjugate design, carrier protein use, and especially adjuvant optimization will significantly enhance the quantity and quality of the antibodies produced, allowing drug vaccines to become useful clinical tools for the treatment of substance abuse (Carfora, 2018).

There is also a considerable knowledge gap regarding ethical issues in vaccines administered during pregnancy. Several crosscutting themes concerning ethics in maternal immunization have emerged. The more critical of these themes are the need for public justification and transparency, proper framing of information, and the importance of ensuring quality routine care delivery with the introduction of new interventions into the antenatal care platform (Beelera, 2016).

\section{References}

Amirthalingam, G., Andrews, N., Campbell, H., Ribeiro, S., Kara, E., Donegan, K., et al. (2014) Effectiveness of maternal pertussis vaccination in England: an observational study, Lancet; 384(9953), pp. 1521-1528, doi: 10.1016/S0140-6736(14)60686-3.

Anderson, T. S., Dave, S., Good, C. B. \& Gellad. W. F. (2014) Academic medical center leadership on pharmaceutical company boards of directors, JAMA, 311(13), pp. 1353-1355, doi: 10.1001/jama.2013.284925.

Baker, C. J. \& Kasper, D. L. (1976) Correlation of maternal antibody deficiency with susceptibility to neonatal group B streptococcal infection, The New England Journal of Medicine, 294(14), pp. 753-756.

Bremer, P. T., Schlosburg, J. E., Banks, M. L., Steele, F. F., Zhou, B., Poklis, J. L. \& Janda, K. D. (2017) Development of a clinically-viable heroin vaccine, Journal of the American Chemical Society, 139(25), pp. 8601-8611, doi: 10.1021/jacs.7b03334.

Berkowitz, B. \& Spector, S. (1972) Evidence for active immunity to morphine in mice, Science, 178(4067), pp. 1290-1292, doi: 10.1126/science.178.4067.1290.

Bonese, K. F., Wainer, B. H., Fitch, F. W., Rothberg, R. M. \& Schuster, C. R. (1974) Changes in heroin self-administration by a rhesus monkey after morphine immunization, Nature, 252, pp. 708-710, doi:10.1038/252708a0.

Beelera, J, A., Lambach, P., Fulton, T. R., Narayanan, D., Ortiz, J. R. \& Omer, S. B. (2016) A systematic review of ethical issues in vaccine studies involving pregnant women, Human vaccines \& immunotherapeutics, 12(8), pp. 1952-1959, doi: $10.1080 / 21645515.2016 .1186312$. 
A. Ihan: Challenges posed by novel vaccines in a hyperlinked society both to doctors and the health of their patients

Carfora, A., Cassandro, P., Feola, A., La Sala, F., Petrella, R. \& Borriello, R. (2018) Ethical Implications in Vaccine Pharmacotherapy for Treatment and Prevention of Drug of Abuse dependence, Journal of Bioethical Inqueries, 15(1), pp. 45-55, doi: 10.1007/s11673-017-9834-5.

Haney, M., Gunderson, E. W., Jiang, H., Collins, E. D. \& Foltin, R. W. (2010) Cocainespecific antibodies blunt the subjective effects of smoked cocaine in humans, Biological Psychiatry, 67(1), pp. 59-65, doi: 10.1016/j.biopsych.2009.08.031..

Hawkins, B., Holden, C. \& McCambridge, J. (2012) Alcohol industry influence on UK alcohol policy: a new research agenda for public health, Critical Public Health, 22(3), pp. 297-305, doi: 10.1080/09581596.2012.658027.

Leask, J. (2013) Pregnant women's intention to take up a postpartum pertussis vaccine, and their willingness to take up the vaccine while pregnant: a cross sectional survey, Vaccine, 31(37), pp. 3972-3978, doi: 10.1016/j.vaccine.2013.06.015.

Lancefield, R. C. (1934) A Serological differentiation of specific types of bovine hemolytic streptococci (group B), Journal of Experimental Medicine, 59(4), pp. 441-458.

Nestle, M. (2016) Corporate funding of food and nutrition research: science or marketing? JAMA Internal Medicine, 76(1), pp. 13-14, doi: 10.1001/jamainternmed.2015.6667.

Osburn, A. (2008) Immunizing against Addiction: The Argument for Incorporating Emerging Anti-Addiction Vaccines into Existing Compulsory Immunization Statutes, Cleveland State Law Review, 56(159), pp. 174-185.

Perkmann, M., Tartari, V., McKelvey, M., Autio, E., Broström, A., D’Este, P., et al. (2013) Academic engagement and commercialisation: A review of the literature on university - industry relations, Research Policy, 42(2), pp. 423-442, doi: 10.1016/j.respol.2012.09.007

Polosa, R. \& Benowitz, N. L. (2011) Treatment of nicotine addiction: present therapeutic options and pipeline developments, Trends in Pharmacological Sciences, 32(5), pp. 281-289, doi: 10.1016/j.tips.2010.12.008.

Regan, A. K., Mak, D. B., Hauck, Y. L., Gibbs, R., Tracey, L. \& Effler, P. V. (2016) Trends in seasonal influenza vaccine uptake during pregnancy in Western Australia: implications for midwives, Women and Birth: journal of the Australian College of Midwives, 29(5), pp. 423-429. doi: 10.1016/j.wombi.2016.01.009.

Speed, E. \& Mannion, R. (2017) The rise of post-truth populism in pluralist liberal democracies: challenges for health policy, International Journal of Health Policy and Management, 6(5), pp. 249-251, doi: 10.15171/IJHPM.2017.19

Stowe, G. N., Vendruscolo, L. F., Edwards, S., Schlosburg, J. E., Misra, K. K., Schulteis, G., Mayorov, A. V., Zakhari, J. S., Koob, G. F. \& Janda, K. D. (2011) A Vaccine Strategy that Induces Protective Immunity against Heroin, Journal of Medicinal Chemistry, 54(14), pp. 5195-5204, doi: 10.1021/jm200461m.

Studdert, D. M., Mello, M. M. \& Brennan, T. A. (2004) Financial Conflicts of Interest in Physicians' Relationships with the Pharmaceutical Industry-Self-Regulation in the Shadow of Federal Prosecution, The New England Journal of Medicine, 351(18), pp. 1891-1900, doi: 10.1056/NEJMlim042229.

Shen, X., Orson, F. M. \& Kosten, T. R. (2011) Anti-addiction vaccines, F1000 Medicine Reports, (3), pp. 3-20, doi: 10.3410/M3-20

Volkow, N. D. \& Collins, F. S. (2017) The role of science in addressing the opioid crisis, The New England Journal of Medicine, 377, pp. 391-394, doi: 10.1056/NEJMsr1706626. 